\title{
La programación para niños: perspectivas de abordaje desde el pensamiento lógico matemático
}

Programming for children: approach perspectives from mathematical logical thinking

Jesús Antonio Cerón Molina ${ }^{1}$

Universidad de la Salle

Recibido: 28.08 .2021

Aceptado: 30.10 .2021

\section{Resumen}

El presente artículo hace parte de la investigación "El desarrollo del pensamiento lógicomatemático en la programación para niños: orientaciones pedagógicas basadas en las competencias del siglo XXI", la cual se está desarrollando en el marco del Doctorado en Educación y Sociedad en la Universidad de La Salle. Se presenta un estado del arte cuyo objetivo es identificar los principales avances teóricos que se han presentado en relación con la enseñanza de las matemáticas en básica primaria, las aplicaciones que tiene el lenguaje de programación en los entornos escolares para el desarrollo del pensamiento lógico y el uso de las competencias del siglo XXI como mediador en los procesos de aprendizaje. La investigación se aborda desde un enfoque cualitativo e interpretativo, se hace una revisión de las principales bases de datos como Ebsco, Scopus, Taylor \& Francis, entre otras. Se identifican 60 textos los cuales son estudiados de forma intratextual e intertextual, posibilitando la emergencia de las tres categorías que brindan soporte al marco teórico. Como

\footnotetext{
1 jesusc65@unisalle.edu.co https://orcid.org/0000-0002-4320-2820

Volumen 2. Número 1. Enero - Junio 2022 ISSN: 2745-0341 (En línea)
} 
resultado, se identifica que hay una ausencia de propuestas direccionadas al desarrollo del pensamiento lógico matemático en el lenguaje de programación de dispositivos y falta de propuestas didácticas en la población de básica primaria en relación con la enseñanza de esta área disciplinar.

Palabras clave: Pensamiento lógico matemático, programación para niños y competencias en el siglo XXI.

\section{Abstract}

This article is part of the research "The development of logical-mathematical thinking in programming for children: pedagogical orientations based on the competencies of the XXI century", which is being developed within the framework of the Doctorate in Education and Society at the University of La Salle. A state of the art is presented whose objective is to identify the main theoretical advances that have been presented in relation to the teaching of mathematics in elementary school, the applications that the programming language has in school environments for the development of logical thinking and use of 21 st century skills as a mediator in learning processes. The research is approached from a qualitative approach, a review is made of the main databases such as Ebsco Host, Scopus, Eureka, among others. 60 texts are identified which are studied in an intratextual and intertextual way, allowing the emergence of the three categories that provide support to the theoretical framework. As a result, it is identified that there is an absence of proposals aimed at the development of mathematical logical thinking in the programming language of devices and a lack of didactic proposals in the population of elementary school in relation to the teaching of this disciplinary area.

Keywords: Mathematical logical thinking, programming for children and competences in the 21 st century. 


\section{Introducción}

Considerando la emergencia que han tenido las nuevas tecnologías, la amplia necesidad de pensar el mundo desde la programación de dispositivos y la importancia que tiene el desarrollo del pensamiento lógico matemático se realiza un estado del arte como parte de la investigación doctoral en proceso denominada "El desarrollo del pensamiento lógico-matemático en la programación para niños: orientaciones pedagógicas basadas en las competencias del siglo XXI".

En esta investigación de tipo documental, se establecen tres categorías de análisis: la primera, relacionada con el desarrollo del pensamiento lógico matemático; la segunda, enfocada al estudio de la programación para niños y su avance tanto mundial como nacional y en la tercera, se hace alusión a las competencias en el siglo XXI en una sociedad en constante evolución, en donde la tecnología juega un papel crucial (Martínez, et al., 2021), por tanto, es necesario que la escuela también se transforme con nuevas propuestas a nivel pedagógico y didáctico y de esta manera contribuir a la formación de los estudiantes para enfrentar los retos que este siglo conlleva (Erstad, et al., 2021). En cuanto al aprendizaje de las matemáticas, por su complejidad y su carácter abstracto, un alto porcentaje de los estudiantes la considera compleja y difícil de abordar, de aquí la necesidad de buscar alternativas como la programación desde la niñez, ya que los niños son considerados nativos digitales y presentan facilidad en el uso de dispositivos tecnológicos con los cuales, además de aprender pueden divertirse, todo en el marco de sus intereses (Terroba, et al., 2021).

Desde la mirada de Ramos y Carbonell (2021), la programación es considerada como una de las dimensiones en las que debe enfocarse la escuela actual, por su trascendencia en las profesiones del futuro cercano $\mathrm{y}$, por ende, en los trabajos que emergen del desarrollo tecnológico de la mencionada sociedad del conocimiento, ya que, presentan estrecha relación con el aprendizaje de las matemáticas, sin embargo, en las aulas esta interrelación no se ha logrado establecer de manera efectiva. A nivel mundial esta alternativa ha tenido repercusión en algunos países especialmente europeos, en Latinoamérica los avances son mínimos, 
algunos autores lo atribuyen a la falta de voluntad política, otros a la complejidad de las transformaciones a nivel curricular, formación docente y dotación de instituciones para tal fin. Colombia es uno de los países en los cuales el proceso avanza de forma lenta, se observa el desarrollo de cursos de capacitación a docentes del sector público por parte del Ministerio de Educación en alianza con algunas entidades, pero en la formación del profesorado en programas universitarios que se enfoquen en la didáctica para la programación en los niños, aún no se evidencia un panorama claro sobre esta alternativa.

Un aspecto que es claro, para Cóndor (2020), es el uso exponencial de la tecnología en el ámbito educativo a raíz de la pandemia del Covid-19 en todo el mundo y Colombia no ha sido ajena a involucrar dispositivos en todos los niveles educativos, esto permitió el acceso de la mayoría de la población estudiantil a los recursos tecnológicos, con lo cual se podría abrir la puerta al desarrollo del pensamiento lógico matemático por medio de la programación para niños y de esta forma promover las competencias necesarias para el siglo XXI.

\section{Metodología}

La investigación se llevó a cabo por medio de un diseño cualitativo e interpretativo de tipo documental, para lo cual, se diseñó una matriz bibliográfica en el programa de Excel, en donde se reseñaron 654 documentos: artículos de revista, libros, tesis de maestría y doctorado, entre otros. Mediante el uso de los filtros de Excel se depura la cantidad de documentos y se analizaron: 49 artículos, 5 libros y 6 trabajos de posgrado. Para el correspondiente análisis de los documentos seleccionados se diseñó una matriz analítica de contenido, en el mismo programa mencionado y en la cual se relacionaron los textos, consignados en vertical y las categorías de análisis escritos en horizontal, lo cual facilitó la lectura lineal y transversal de la matriz, para identificar la calidad de la información sobre el tema de investigación y a su vez, detectar los vacíos, repeticiones, confirmaciones y ampliaciones, que sirvieron de insumo en la investigación propuesta y selección de cada una de las categorías ya mencionadas, en donde se planteó: el concepto principal, luego, su estado a nivel mundial, a nivel Latinoamericano y por último, los avances que se han logrado a nivel colombiano. 


\section{Resultados}

Mediante el análisis de las categorías, que proporcionaron el insumo principal del diseño cualitativo e interpretativo de tipo documental, se pueden establecer los siguientes aspectos:

\subsection{El desarrollo del pensamiento lógico matemático: una mirada desde las apuestas de enseñanza.}

Para tal fin, el desarrollo del pensamiento lógico matemático adquiere singular importancia, según Pérez (2019), las matemáticas son la base de las sociedades que han tenido un notable desarrollo tecnológico, entre ellas se destaca china, Corea del Sur, Singapur, Japón, entre otras. Para Nieves, et al (2019), Navegar en el campo de las matemáticas es atender a las leyes de la lógica, en donde se debe privilegiar aspectos relacionados a representar, abstraer, crear y demostrar, para facilitar su correcto aprendizaje. Arias y García (2016), definen el pensamiento lógico matemático como la habilidad con la que cuenta el ser humano para analizar, comprender y resolver problemas de carácter matemático, para Gardner (1998), la inteligencia lógico matemática, está ligada a la resolución de problemas en un determinado contexto, según Piaget (1964), es uno de los tres tipos de conocimiento, en donde se destacan las experiencias y la manipulación de objetos con los que interactúa el niño, para construir su conocimiento desde lo más simple a lo más complejo, ya que este no depende directamente de los objetos, sino de la experiencia que el niño tenga con estos (Castiblanco, 2019).

Aunque el desarrollo del pensamiento lógico matemático no es un tema reciente, es necesario en la evolución de una sociedad que quiera estar a la vanguardia a nivel mundial, por esto, se hace una actualización de los estudios realizados en los últimos años sobre el tema. Valbuena y Alvarado (2020), describen la incidencia de la interactividad en el desarrollo del pensamiento lógico matemático, en donde, los recursos tecnológicos han sido asociados al desarrollo de este campo por medio de un enfoque cualitativo los autores observan e interpretan los resultados de las entrevistas y cuestionarios aplicados a una poblacion de 41 estudiantes, en cinco fases: análisis del papel de los actores educativos, proyectar propuestas 
según el análisis, crear contenidos, puesta en práctica de la acción formativa y por último la evaluación. El estudio reveló que al articular las herramientas tecnológicas al proceso, proporciona resultados fructíferos. A su vez, recomiendan evaluar de forma constante las estrategias metodológicas de los docentes en el area de matemáticas, los cuales reconocen la importancia de desarrollar el pensamiento lógico matemático, sin embargo, sus acciones pedagógicas y didácticas contribuyen muy poco al desarrollo de este aspecto (Del Rosario, et al., 2021). Del mismo modo, Quintanilla (2020), asegura que los docentes reconocen que el desarrollo del pensamiento lógico matemático debe enseñarse desde la didáctica del juego en su parte práctica y en algunos casos el docente conoce las estrategias pero no las aplica, en otros casos se carece del conocimieto necesario.

En este sentido, la capacitacion docente es indispensable, con el fin de no instituir errores en el desarrollo del pensamiento lógico matemático y dominar metodologías dinámicas que capturen la atención e interés del estudiante por este campo considerado complejo y abstracto. Tapia, et al (2020), buscó en su estudio concienciar a los docentes sobre la importancia del desarrollo del pensamiento lógico en el área de matemáticas, por medio de la estrategia medotológica del Aprendizaje Basado en Proyectos (ABP). Se llega a la conclusión que el ABP no solo desarrolla el pensamiento lógico matemático, sino también el pensamiento crítico y reflexivo. Suñe (2019), estudia la habilidad que tienen los futuros docentes para desarrollar el pensamiento lógico matemático en niños y con esto analizar los errores que se presentan. Los estudiantes de licenciatura en matemáticas evidencian poca apropiación en cuestiones relacionadas al uso de la didáctica para desarrollar el aspecto lógico por medio de la búsqueda de atributos de diferentes objetos para formar proposiciones falsas y verdaderas. Según el estudio la principal dificultad radicó en las instrucciones que ofrecía el docente, las cuales no permitían que el estudiante lograra descubrir los conceptos trabajados. Se concluye que la población estudiada no cuenta con el rigor que requiere el campo matemático para un correcto desarrollo de la lógica en los niños, este aspecto tiende a perjudicar a la población de infantes que reciben formación con docentes que no cuentan con las herramientas apropiadas y permite observar que el sistema educativo carece de estrategias para detectar estos casos en un área fundamental no solo para el crecimiento personal de los niños sino de toda la sociedad. 
Los estudios no solo atienden las dificultades presentadas por los docentes como aspecto fundamental en el desarrollo del pensamiento lógico matemático, también son relevantes las dificultades de los estudiantes, como es el caso de la investigación realizada por Sánchez y García (2021), quienes se propusieron diseñar un protocolo con el cual los niños pudieran superar dificultades como la discalculia, según Montoya (2017), es un trastorno del aprendizaje en donde el niño presenta dificultad en cuanto al uso de los números, por ende se ve afectada su capacidad para resolver problemas aritméticos, dado que esto le impide razonar de forma lógica y operar con símbolos matemáticos. La investigación utilizó la prueba denominada TEDI-MATH, en la cual está compuesta por subpruebas que se enfocan en aspectos claves de las matemáticas como: contar, numerar, comprender el sistema numérico, desarrollar operaciones lógicas, estimar el tamaño entre otros. La población fue dividida en grupo de control y experimental, el último grupo por medio del protocolo logró mejorar la comprensión del sistema numérico. Para Arizaga y Román (2021), la respuesta está en la “resolución de problemas matemáticos que permiten menguar los efectos de este trastorno, toda vez que desarrolla las habilidades y competencias matemáticas” (p.442).

Las investigaciones consultadas, permiten observar una tendencia en la actualidad con relación al uso de la neurociencia en el desarrollo del pensamiento lógico matemático en general, para Pacheco (2013), Guerra (2019) y Castillo (2019), estudiar la estructura y función del cerebro en los niños permite establecer estrategías que conllevan a un mejor aprendizaje. Según los resultados de las investigaciones es en la niñez en donde se produce la mayor cantidad de mielina, sustancia que favorece la transmición de impulsos eléctricos a lo largo de las neuronas, su produccción esta dada según los estímulos sensoriales que recibe cada individuo en su entorno, de ahí la importancia de conocer estos aspectos en el sistema educativo, con el ánimo de establecer didácticas que fovarezcan el desarrollo cerebral, en el caso del pensamiento lógico matemático se debe estimular el cuadrante A en el lobulo superior izquierdo del cerebro. 
Para Ocampo, et al (2020), por medio de los videojuegos se activan receptores sensoriales que estimulan el cerebro y su estudio se enfocó en establecer si los videojuegos contribuyen al desarrollo del pensamiento lógico matemático en los niños, para lo cual estructuraron cinco interronantes de investigación que responden al método de Torres-Carrión, el cual propone una metodológia sistemática de busqueda de literatura en tres fases: Planificación, desarrollo de un protocolo de revisión y realización de la revisión. Entre las conclusiones de este estudio se destaca: para que se obtengan resultados exitosos debe estar clara la coherencia entre los métodos pedagógicos y las herramientas tecnologicas que utilizan los niños, no todos los videojuegos desarrollan el pensamiento lógico matemático. Host'ovecký, et al (2018), reconocen que los niños tienen gran interés en los videojuegos por lo cual, se han desarrollado diferentes propuestas educativas en este campo, con la finalidad de hacer más llamativas materias como las matemáticas.

Existen diversas formas para desarrollar el pensamiento lógico matemático en los estudiantes básica primaria y esto según Castiblanco (2019), proporciona al individuo herramientas de formación desde la infancia y que le serán útiles a corto, mediano y largo plazo tanto en la parte personal como social. Para el docente investigador el reto en la actualidad consiste en construir estratégias mediante las cuales el estudiante desarrolle su pensamiento lógico matemático y este motivado a construir conceptos mientras se divierte aplicando las matemáticas. Entre las estrategias que estan siendo incrementadas en las aulas de clase desde la primera infancia y que constiyuye un verdadero reto por la innovación que conlleva en Colombia, es la programación para niños, la cual esta enmarcada en la segunda categoria a desarrollar

\subsection{La programación en la educación primaria: retos y desafíos}

Este aspecto a nivel mundial no es nuevo, algunos países como: Inglaterra, Corea del Sur, suecia, Finlandia, Macedónia entre otros, tienen en sus currículos de forma obligatoria la programación para los niños, lo cual conlleva que sus docentes adquieran formación especializada sobre el tema y la transferencia de conocimientos sea éxitosa (Llorens, et al., 
2017). Para Fatourou, et al (2021), se terminara adoptando la programación como una de las materias esenciales en los sistemas educativos en todos los niveles de escolaridad. En America Latina, la programación para niños en los planes de estudio tiene un proceso insipiente y en la mayoría de los países depende de los gobernantes de turno, por que la educación no es considerada una política de estado, sino una política pública.

Los procesos en este aspecto muestran esfuerzos aislados, según Casali, et al (2020), el Consejo Federal Argentino "en septiembre de 2018 se aprobaron los "Núcleos de Aprendizajes Prioritarios de Educación Digital, Programación y Robótica" (NAP EDPR) para los diferentes niveles de la educación obligatoria (Res. CFE No 343/18, 2018)” (p.596). Para este fin, se estableció un plazo de dos años, en los cuales se debía modificar planes de estudio, capacitar docentes y establecer convenios con universidades para crear programas, por ejemplo "Especialización Docente de Nivel Superior en Didáctica de las Ciencias de la Computación: Aprendizaje y Enseñanza del Pensamiento Computacional y la Programación en el Nivel Primario" (p.56).

En el ámbito colombiano, se han presentado diferentes convenios para crear estrategias que lleven el pensamiento computacional y la programación a los diferentes niveles educativos, pero sin mayores cambios en la parte curricular. El último convenio registrado esta desde el año 2019, entre el Ministerio de Tecnologías de la información y las Comunicaciones (MINTIC), Ministerio de Educación nacional (MEN) y el British Council (BC), para llevar a cabo un programa de formación para docentes en programación para niños y niñas en el sector oficial, una las condiciones para acceder al curso es la transferencia a la mayor cantidad posible de estudiantes. Entre las metas esta la creación de comunidades de aprendizaje, en donde el docente pueda compartir y aprender de sus colegas para que transforme su práctica pedagógica e integre la programación a su aula.

Articular el pensamiento computacional (PC), terminología de Wing (2006), para referirse al uso de la informática en la solución de problemas, utilizando un agente de procesamiento de la información. Según el autor, el PC, es una habilidad que debe ser desarrollada por todos, 
así como la lectura o la escritura, en este sentido, Zapata (2015) y García, et al (2018), hablan de una alfabetización en este campo del conocimiento, como una de las premisas fundamentales para los ciudadanos del siglo XXI, en donde no solo se sean consumidores de programas tecnológicos y se conviertan en productores de sus propios contenidos.

Aprender a programar para Gómez (2020), es similar a aprender un nuevo idioma, es necesario aprender nuevo vocabulario y conceptos, con los cuales interactuar en este caso con el computador o dispositivo tecnológico y comunicar las instrucciones de tal forma que la máquina entienda la información y la procese según los requerimientos establecidos. La programación para niños al ser una disciplina nueva, no cuenta con grandes estudios de investigación relacionados a su didáctica, por lo cual el autor diseñó, un entorno para favorecer la expresividad en el lenguaje, para detectar errores y proponer alternativas o métodos para adquirir el lenguaje de programación basado en el aprendizaje del lenguaje natural, por lo cual, asegura que es posible enseñar lenguaje de programación a niños de cualquier edad, incluso si aún no saben leer y escribir.

Acuña, et al (2018), Durango y Ravelo (2020) y Franco, et al (2020), proponen el uso del programa Scratch, para aprender a programar con un lenguaje sencillo, adaptado a los niños, en donde pueden desarrollar la creatividad, solucionar problemas de manera interactiva y trabajar en equipo, por tanto, se propone que se trabaje de forma transversal en el currículo este tipo de lenguaje por medio de proyectos para que el estudiante construya su propio conocimiento apoyado en el entorno y en el lenguaje de programación. Otro recurso utilizado para enseñar a programar a los niños son los robots, los estudios de Caballero y García (2021), como los de López y Yuste (2017), demostraron que estos elementos fomentan las habilidades en cuanto a la programación y al pensamiento computacional en general al programar emociones a un robot, para lo cual, el niño debe gestionar sus propias emociones y con esto poder lograr tener un desarrollo cognitivo emocional mayor al tiempo que interactua con la programación como parte de su desarrollo integral. 
En este sentido, para el desarrollo de la programación en los niños, la mayoría de sistemas educativos, no están preparados para asumir todo lo que esto conlleva, no obstante, según Moura, et al (2021), el COVID-19, transformó la educación desencadenando que docentes, estudiantes y padres de familia adquirieran competencias digitales, con el fin de minimizar los impactos desfavorables que trajo el confinamiento, con esto, las familias adquirieron dispositivos tecnológicos y conexión a internet como un aspecto básico en la educación, aspecto que pudiese favorecer la proliferación de la programación en la infancia. Basado en este panorama y considerando las categorías del desarrollo del pensamiento lógico matemático y la programación para niños se expone la siguiente categoría, la cual está relacionada con las competencias necesarias para el siglo XXI.

\subsection{Competencias del siglo XXI: una apuesta por la resolución de problemas}

Según la Organización para la Cooperación y el Desarrollo Económico OCDE (2012), este tipo de competencias representan el conjunto de conocimientos, habilidades y destrezas con las que un individuo debe afrontar los retos actuales y en el futuro. Para la Organización de las Naciones Unidas para la Educación, la Ciencia y la Cultura UNESCO (2015), como la OCDE (2018), es indispensable el desarrollo de estas competencias desde la infancia y fomentar en los estudiantes habilidades que les conduzcan a la solución de problemas de su entorno de manera crítica y responsable con el uso de la tecnología como una de las principales herramientas del siglo XXI. Las competencias necesarias en el siglo XXI son 10 y están organizadas en 4 categorías, Binkley et al (2012), las presenta de la siguiente manera: Maneras de pensar: creatividad e innovación, pensamiento crítico, resolución de problemas y aprender a aprender. Herramientas para trabajar: apropiación de las tecnologías digitales y manejo de la información. Maneras de trabajar: comunicación y colaboración. Maneras de vivir en el mundo: vida y carrera, responsabilidad personal y social y ciudadanía local y global.

En cuanto a esta investigación, se asume la categoría número uno, la cual está enfocada en la resolución de problemas como una de las alternativas para formar a los estudiantes desde la infancia para enfrentar y solucionar los problemas propios de las sociedades globalizadas y 
matematizadas por la tecnología, (Gutiérrez et al., 2017), en esta línea tenemos los siguientes autores. Por medio de la tecnología y en particular los videojuegos, Ríos y Díez (2018), desarrollaron una propuesta para entornos multigrado, enfocados en "habilidades sociales, habilidades para la resolución de problemas, planificación de estrategias, toma de decisiones, búsqueda de información, colaboración en grupo, descubrimiento inductivo, razonamiento lógico, autocontrol" (p.8). El docente que tiene a su cargo varios cursos en un solo espacio debe ser innovador y formar a sus estudiantes para la actualidad, por lo cual se llevó a los docentes a reflexionar sobre su práctica y los beneficios que tienen los videojuegos en el desarrollo de competencias necesarias en el siglo XXI.

Otros aspectos que influyen en la adquisición de competencias según Castro et al. (2018), es el género, la formación académica de los padres y la cantidad de libros leídos en un determinado hogar, adicional a esto, en cuanto a las instituciones, los autores resaltan: la cantidad de estudiantes que conviven en un aula escolar, la calidad y cantidad de los materiales didácticos para su aprendizaje, el tipo de colegio y la autonomía que este tiene para desarrollar proyectos que fortalezcan las competencias de sus estudiantes. Giménez et al. (2019), Sartor, et at. (2020), y Ponce et al. (2019), añaden como aspectos fundamentales, el ambiente escolar, la formación docente y el desarrollo de sus competencias como base para formar competencias en los estudiantes.

En Colombia, una de las evaluaciones de carácter internacional es llevada a cabo por el Programme for International Student Assessment (PISA) que es desarrollada por la OCDE para evaluar las competencias de la población estudiantil de 15 años de los países que la componen; entre las competencias se hace relación a la resolución de problemas como como parte esencial de la prueba. Según Según la Organización para la Cooperación y el Desarrollo Económico OECD (2019), al analizar los resultados de la prueba elaborada en el 2018, el 99\% de los estudiantes presentó dificultad en el nivel 5, en donde se modelan, comparan y evalúan estrategias para solucionar problemas. A nivel nacional, la evaluación que desarrolla el Instituto Colombiano para la Evaluación de la Educación (ICFES), el 95\% de los estudiantes presentaron dificultad para solucionar el nivel 4, que es el nivel superior de esta prueba en el 
campo de las matemáticas y está orientada en la solución de situaciones problemas, en donde se debe: interpretar, representar, formular, ejecutar y argumentar, (ICFES, 2021). La problemática es general, sin embargo, para Cruz, et al (2020) y Lorduy (2021), los resultados al ser comparados entre colegios privados y públicos, los primeros mostraron mejor nivel frente a los segundos, ubicandose en los primeros lugares de la prueba.

\section{Discusión}

Como docente de básica primaria del sector oficial en Colombia, preocupado por el fomento de las habilidades y competencias de los niños y niñas, con las cuales puedan aportar al desarrollo social del pais en el siglo XXI, propendo por una educación que este basada en la necesidades de la comunidad local, nacional e internacional, por lo cual, al analizar la primera categoria propuesta en este estudio, emergen aspectos a considerar:

. Las pruebas que evaluan el pensamiento lógico matemático, entre ellas: PISA y la prueba Saber Pro, desarrolladas por OCDE (2018) e ICFES (2020), respectivamente, año tras año muestran grandes dificultades en este campo del saber, sin que se establezcan mayores medidas que conlleven a superarlas desde el trabajo educativo infantil.

- El rezago en las instituciones públicas en cuanto a la apropiacion de la tecnología como herrameinta didactica, no solo atiende a factores económicos como lo muestran algunos autores, también a tiende a variables tales como: la formación de los docentes, el nivel educactivo de los padres de familia, entre otras.

Con respecto a la programación para niños, la cual compone la segunda categoría, se puede Resaltar:

. En Colombia se debe promover la programación en los niños como una materia del programa curricular por todas las ventajas que conlleva y su facilidad de ser articulada con las cualquier 
materia del currículo, en especial con las matemáticas. Podríamos tomar como referente el proceso del caso Argentino, mensionado en el estudio de (Casali, et al., 2020), claro que, atendiendo a nuestras propias características y condiciones.

. La necesidad de personal idóneo en el campo de la programación y las nuevas tecnologías, ya se comienza a notar en el país, por ende, no solo es un derecho de los ciudadanos de básica primaria formarse como individuos críticos, dotados de herramientas que los lleve a crear tecnología y les permita dejar de ser simples consumidores. Para esto es necesario el aprendizaje del lenguaje de programación, el cual puede ser aprendido desde la infancia sin ningún tipo de requisito especial, (Gómez, 2020).

Colombia, requiere el desarrollo de las competencias de para el siglo XXI, en todos los niveles educativos, pero en especial en los estudiantes en edad infantil, conceptos que componen la tercera categoria del estudio. Según la OCDE (2018), este tipo de competencias se deben desarrollar desde la infancia, sin embargo, al estar ubicados en los últimos lugares de la pruebas internacionacionales, se deduce que este aspecto tan importante para el futuro del país, en la escuela no es prioridad.

\section{Conclusiones}

El pensamiento lógico matemático el cual se centra en la resolucion de problemas es uno de los aspectos relevantes para el desarrollo de competencias necesarias para el siglo XXI, las cuales se fundamentan en el uso de la tecnologia, por tanto, la programación para niños adquiere importancia en gran medida en que sea llevada al aula interrelacionada con el área de matemáticas desde la infancia. Por tanto, algunos sistemas educactivos a nivel mundial ya tienen en sus planes de estudio desde la infancia el aspecto curricular de la programación como uno de los factores para dotar a sus estudiantes de herramientas para afrontar los retos del siglo XXI, solucionar problemas que aun no se han vislumbrado, ya que estaran ejerciendo en 
profesiones que no se han creado en la sociedad actual, pero que seran necesarias para el desarrollo tecnológico y el mejoramiento de la calidad de vida.

En latinoamérica uno de los paises en donde se comienza a evidenciarse cambios en el sector educativo con el uso de la programación desde la niñez es: Argentina, esto ha implicado cambios a nivel curricular, dotación de recursos tecnológicos a las instituciones de educación inicial, básica y media, y la formación docente por medio de convenios con diversas universidades para crear especializaciones y maestrías centradas tanto en la enseñanza como en el aprendizaje de la programación en los niños.

En colombia, los avances son mínimos en este campo, la investigación documental llevada a cabo refleja unicamente la capacitación docente por medio de cursos cortos, en donde los docentes del sector público son formados para la transferencia a sus estudiantes, pero sin seguimiento de personal idóneo en el tema. A pesar de las dificultades, se ha encontrado que algunos docentes dedican sus esfuerzos y recursos en su formación y por ende en la formación de sus estudiantes, con propuestas didácticas y pedagógicas novedosas para motivar los aprendizajes y se constituyan en competencias capaces de transformar la sociedad actual en todos sus aspectos, para consolidar un desarrollo sostenible en el siglo XXI. Basados en estos argumentos, se requiere afianzar el uso de la tecnología para los procesos educativos, en donde toda la comunidad se vio obligada a enfrentar transformaciones escolares y pasar de la modalidad presencial a la remota o virtual, a causa de la pandemia del Covid-19, esto si duda trajo consigo un cambio de paradigma en especial en la educación de los niños y seguramente abrirá espacios para el desarrollo del pensamiento lógico matemático, por medio de la programación, con el fin de fometar las competencias necesarias para el siglo XXI. 


\section{Bibliografía}

Acuña, N., León, M., López, L., Villar, C., \& Mulford, R. (2018). Learning Mathematics MidInteractive. Cultura. Educación y Sociedad, 9(2), 32-42. doi: http://dx.doi.org/10.17981/cultedusoc.9.2.2018.03

Árizaga, A. \& Román, J. (2021). La discalculia en alumnos de la educación básica. Revista Sociedad \& Tecnología, 4(3), 432-446.

Arias, C., y García, L. (2016). Los juegos didácticos y su influencia en el pensamiento lógico matemático en niños de preescolar de la Institución Educativa el jardín de Ibagué [Tesis de Maestría, Universidad Robert Wiener]. http://repositorio.uwiener.edu.pe/handle/123456789/625

Binkley, M., Erstad, O., Hermna, J., Raizen, S., Ripley, M., Miller, M., \& Rumble, M. (2012). Defining 21st century skills. En P.E. Griffin, B. McGaw, \& E. Care (Eds.), Assessment and teaching of 21 st century skills (p.17-66). Dordrecht, Netherland: Springer.

Caballero, Y., \& García, A. (2021). Robots in the Early Childhood Education: learning to sequence actions using programmable robots. RIED. Revista Iberoamericana de Educación a Distancia, 24(1), 77-94. doi: http://dx.doi.org/10.5944/ried.24.1.27508

Casali, A., Deco, C., Viale, P., \& Bender, C. (2020). Enseñanza y aprendizaje del pensamiento computacional y la programación en los distintos niveles educativos. XXII Workshop de Investigadores en Ciencias de la Computación, (págs. 595-599). El Calafate, Santa Cruz. http://sedici.unlp.edu.ar/handle/10915/104106

Castiblanco, E. (2019). Las herramientas interactivas y su relación con el fortalecimiento del pensamiento Logico-matemático en niños. Huellas Rurales, 5(2), 22-36. https://www.revistas.upel.edu.ve/index.php/huellasrurales/article/view/8172/4787 
Castro, G., Giménez, G., \& Pérez, D. (2018). Estimación de los factores condicionantes de la adquisición de competencias académicas en América Latina en presencia de endogeneidad. Revista CEPAL, (124), $35-59$. https://www.cepal.org/sites/default/files/publication/files/43459/RVE124_Castro.pdf

Cóndor, O. (2020). Educar en tiempos de COVID-19. CienciAmérica, 9(2), 31-37. http://201.159.222.118/openjournal/index.php/uti/article/view/281/419

Cruz, A., Hernández, A., \& Silva, A. (2020). Covertura de las TIC en la educación básica rural y urbana en Colombia - 2018. Revista Cientifica Profundidad Construyendo Futuro, 13(13), 39-48. https://revistas.ufps.edu.co/index.php/profundidad/article/view/2578/2657

Del Rosario, J., Díaz, T., Bastardo, E., Cortegaza, L., \& Díaz, M. (2021). Estrategias metodológicas para el desarrollo del pensamiento lógico en los alumnos de sexto grado de Primaria. UCE Ciencia. Revista de postgrado, 9(2), 1-5. http://uceciencia.edu.do/index.php/OJS/article/view/234

Durango, C., \& Ravelo, R. (2020). Benefits of Implementing the Scratch Software to Enhance. $\begin{array}{llll}\text { Trilogía Ciencia Tecnología } & \text { Sociedad, } & \text { 12(23), }\end{array}$ https://doi.org/10.22430/21457778.1524

Erstad, 0., Miño, R., \& Rivera, P. (2021). Prácticas educativas para transformar y conectar escuelas y comunidades. Comunicar, 29(66), 9-20.

Fatourou, E., Zygouri, N., Loukopoulos, T., \& Stamoulis, G. (2021). Review of Learning Design Choices of Primary School Programming Courses in Empirical Researches. 2021 IEEE Global Engineering Education Conference (EDUCON), (págs. 1010-1018). 
Franco, D., García, D., Guevara, C., \& Erazo, J. (2020). Scratch for teaching programming language in the first year of high school. Revista Arbitrada Interdisciplinaria KOINONIA, 5(Especial II), 398-414. http://dx.doi.org/10.35381/r.k.v5i5.1050

García, F. J., \& Méndez, A. J. (2018). Exploring the computational thinking effects in preuniversity education. Computers in Human Behavior, 80, 407-411. https://doi.org/10.1016/j.chb.2017.12.005

Gardner, H. (1998). Intelligence in seven steps. Creating the future: Pers - pectives on educational change. https://cahyadsn.phpindonesia.id/psycho/mi/mi003.pdf

Giménez, G., Barrado, B., \& Arias, R. (2019). El papel del profesorado y el entorno de aprendizaje en el rendimiento de los estudiantes costarricenses: un análisis a partir de PISA. Revista Complutense De Educación, 30(4), 1127-1145. https://doi.org/10.5209/rced.60189

Gómez, M. (2020). Aspectos de adquisición de lenguaje en la enseñanza de programación. [Tesis de doctorado, Universidad Nacional de Córdoba]. Repositorio Institucional. https://www.researchgate.net/publication/345256073

Gutiérrez, R., Prieto, J., \& Ortiz, J. (2017). Matematización y trabajo matemático en la elaboración de simuladores con GeoGebra. Educación matemática, 29(2), 37- 68. https://doi.org/10.24844/em2902.02

Guerra, E. (2019). Enfoques de Neurociencia para mejorar pensamiento lógico-matemático en estudiantes del tercer grado de primaria, Institución Educativa La Inmaculada Huamachuco 2019. [Tesis de doctorado, Universidad Cesar Vallejo]. https://repositorio.ucv.edu.pe/handle/20.500.12692/48577

Host'ovecký, M., Salgovic, I., \& Virage, R. (2018). Serious Game in Science Education: How we can Develop Mathematical Education. 2018 16th International Conference on Emerging 
eLearning Technologies and Applications (ICETA), (págs. 513-531). Stary Smokovec, Slovakia.

ICFES. (2021). Informe nacional de resultados del examen Saber $11^{\circ} 2020$ (vol. I).

López, V., \& Yuste, R. (2017). EMOROBOTIC: Gestión Emocional a través de la Programación en Robots en Educación Primaria. Eindhoven, Propuestas de innovación educativa en la sociedad de la información (pp. 82-91). NL: Adaya Press.

Lorduy, J. (2021). Los mejores colegios según los últimos resultados de la prueba Saber 11 de 2020. La República. https://www.larepublica.co/especiales/los-mejores-colegios-de2020/los-mejores-colegios-segun-las-pruebas-icfes-3135699

Llorens, F., García, f., Molero, X., \& Vendrell, E. (2017). The Teaching of Computer Science, Programming and Computational Thinking in Pre-University Studies. Education in the Knowledge Society, 18(2), 7-17.

Martínez, M., Sábada, C., \& Serrano, J. (2021). Meta-marco de la alfabetización digital: análisis comparado de marcos de competencias del Siglo XXI. Revista Latina de Comunicación Social, 79, 76-110. https://www.doi.org/10.4185/RLCS-2021-1508

Montoya, C. (2017). Aplicación del Programa de Estrategias Lúdicas para superar la Discalculia en los estudiantes del 3 er grado de primaria de la I.E. Juan Pablo II El Agustino, 2016. [Tesis de maestría. Universidad César Vallejo, Perú]. https://hdl.handle.net/20.500.12692/7365

Moura, M., Luderitz, M., \& Réal, J. (2021). El “desierto digital”: repercusiones de la COVID19 en la Educación en España y Brasil. Revista Electrónica Interuniversitaria de Formación del Profesorado, 24(2), 181-191. 
Nieves, S., Caraballo, c., \& Fernández, C. (2019). Methodology for the development of the logical mathematical thought from the demonstration by complete induction. MENDIVE, 17(3), 393-408. http://mendive.upr.edu.cu/index.php/MendiveUPR/article/view/1681

Ocampo, W., Garc, J., Macas, A., Carrasco, F., \& Centeno, J. (2020). Videojuego Educativo para el pensamiento para el desarrollo del pensamiento lógico-matemático en educación básica: Revisón sistemática de literatura. Risti, E(31), 513-531.

OCDE (2018). The future of education and skills. Education 2030. http://go.uv.es/1fDpQnn

OECD (2019), PISA 2018 Results (Volume I): What Students Know and Can Do, PISA, OECD Publishing, Paris. https://doi.org/10.1787/5f07c754-en

Pacheco, G. (2013). ¿Qué es la neurociencia? [mensaje en un blog]. Blog Teorías de la neurociencia. http://teoriasneurocienciaequipocuatrosaia.blogspot.com/

Pérez, M. (2019). Efecto del método de resolución de problemas en el logro de la competencia matemática del $4^{\circ}$ grado del ciclo avanzado en el CEBA $N^{\circ} 109$ Inca Manco Capac UGEL 05 San Juan de Lurigancho, 2015. [Tesis doctoral, Universidad Nacional de Educación Enrique Guzmán y Valle]. https://repositorio.une.edu.pe/handle/UNE/4205

Piaget, J. (1964). Part I: Cognitive development in children: Piaget / Development and learning. Journal of Research in Science Teaching, 2(3), 176-186.

Ponce, N., \& Camus, P. (2019). La Práctica como eje Formativo-Reflexivo de la Formación Inicial Docente. Revista de estudios y experiencias en educación, 18(37), 113- 128. https://dx.doi.org/10.21703/rexe.20191837ponce1 
Quintanilla, N. (2020). Playful strategies for teaching mathematics at the nivel of Primary $\begin{array}{llllll}\text { Education. } & \text { Mérito, } & 2(6), & 143 & - & 157 .\end{array}$ https://revistamerito.org/index.php/merito/article/view/261

Ramos, Y., \& Carbonell, Y. (2021). ¿Por qué no estudiar matemáticas? EduSol, 21(74), 218229.

Ríos, M., \& Díez, N. (2018). Políticas de mejora continua. garantía de la calidad en centros de educación superior. Revista Panorama, 12(23), http://dx.doi.org/10.15765/pnrm.v12i23.1191

Sartor, A., Azevedo, J., Pueyo, S., \& Tejedor, S. (2020). Análisis de las competencias docentes en proyectos de aprendizaje-servicio en la educación superior: La percepción del profesorado. Formación universitaria, 13(3), 31-42. https://dx.doi.org/10.4067/S071850062020000300031

Suñe, M. (2019). Importance of the logical mathematical competence in students of the Degree in Early Childhood Education. Números, 103, 49-64. http://www.sinewton.org/numeros

Tapia, Y., Garcia, D., Erazo, J., \& Narváez, C. (2020). Problem-Based Learning as a didactic strategy for the development of. Revista Arbitrada Interdisciplinaria KOINONIA, 5(1), 753 772. http://dx.doi.org/10.35381/r.k.v5i1.808

Terroba, M., Rivera, J., \& Lapresa, D. (2021). cultivando el talento matemático en educación infantil mediante la resolución de problemas para favorecer el desarrollo del pensamiento computacional. Contextos educativos, (28), 65-85.

UNESCO (2015). Replantear la educación. ¿Hacia un bien común mundial? Paris, France: UNESCO. 
Wing, J. (2006). Computational thinking. Communications of the ACM. 49(2), 33- 35. doi:10.1145/1118178.1118215.

Zapata, M. (2015). Pensamiento computacional: Una nueva alfabetización digital. RED, 46, 1-47. https:// doi.org/10.13140/RG.2.1.3395.8883 